\title{
Patient satisfaction, one of the levers for the health services performance: Contribution to scientific evidence, Lubumbashi, Democratic Republic of Congo
}

\author{
Satisfaction des patients, l'un des leviers pour la performance des services de santé : Contribution à l'évidence \\ scientifique, Lubumbashi, République Démocratique du Congo
}

\author{
Adrien Malandj Kes ${ }^{1}$, Nonon Mulubwa Kyalubile ${ }^{3}$, Mujinga Kashala ${ }^{4}$, Katshiza Tshisand ${ }^{2}$, Henri Mundongo \\ Tshamba $^{1,2^{*}}$, Andre Ndala Nyiongonyi ${ }^{1}$, Françoise Malonga Kaj ${ }^{1,2}$ \\ ${ }^{1}$ University of Lubumbashi, School of Public Health, Lubumbashi, RDC \\ ${ }^{2}$ University of Lubumbashi, Faculty of Medicine; Lubumbashi, RDC \\ ${ }^{3}$ Institut Supérieur des Techniques Médicales de Lubumbashi, RDC \\ ${ }^{4}$ Institut Supérieur des Techniques Médicales de Kolwezi, RDC
}

Received 12 July 2018, Accepted 15 Sept 2018, Available online 20 Sept 2018, Vol.6 (Sept/Oct 2018 issue)

\begin{abstract}
The study objectives were to determine the level of patient's satisfaction, to evaluate the health care quality, to identify factors associated with the satisfaction and the impact on the hospital performance. It was conducted between 01-July2016 and 01-July-2017, in Lubumbashi city. This is a cross-sectional study including 423 patients. The interview using a questionnaire with the Likert scale and direct observation allowed the data collection. Analyses were performed using Excel 2013, EPI INFO 7 and SPSS 21. The Student $t$ test, the Levene test and the Khi square test allowed comparison of means, variances and proportions. The odds ratio was calculated for some variables and the significance level set at $p \leq$ 0.05 and $95 \% \mathrm{Cl}$. The analysis of the validity and reliability of the questionnaire made by calculating the Cronbach $\alpha$ coefficient, the number of dimensions of the questionnaire with a principal component analysis (PCA), the correlation coefficient, the Bartlett test and the measurement accuracy of the Kaiser-Meyer-Olkin sample were calculated. The study results show that $6 \%$ of hospitalized patients were very satisfied and only $47 \%$ satisfied. Patients who felt they were well received, were satisfied overall with $56 \%$ and $47 \%$ of the patients who judged the reception and the rooms comfort of poor quality at the first contact declared a dissatisfaction. This observation indicates that the first contact is crucial for patients. Moreover, we observed that more than $70 \%$ of the satisfied patients, the organizational aspects the waiting line management reducing the wait time, the communication between patient and heath staff on the therapeutic approach, the patient participation in the care process and decision, individualization of care were crucial ( $p$ $=0.000)$. In addition, the policy of quality management has led to costs and price increases, but on the other hand, the care demands by patients have remained insensitive to this increase in costs and the level of satisfaction quite high for patients who have benefited care in these services $(p=0.000)$. The survey reveals that quality for the patient is a concept related to his satisfaction. Quality management provides a competitive advantage for healthcare institutions and improves the level of patient satisfaction and services utilization, one of the levers of health service performance.
\end{abstract}

Keywords: Satisfaction, Patients, Performance Lever, health services, Lubumbashi, DRC

\section{Résumé}

L'étude avait pour objectifs de déterminer le niveau de satisfaction des patients hospitalisés, évaluer la qualité des soins, identifier les déterminants de la satisfaction et l'impact sur la performance des services hospitaliers. Elle a été réalisée entre le 01-Juillet-2016 et le 01-Juillet-2017, à Lubumbashi. C'est une étude transversale, menée auprès de 423 patients. L'interview par un questionnaire utilisant l'échelle de Likert et l'observation directe ont permis la collecte des données, analysées en utilisant Excel 2013, EPI INFO 7 et SPSS 21. Le test $t$ Student, le test de Levene et le test de Chi ${ }^{2}$ ont permis la comparaison des moyennes, des variances et les proportions. L'Odds ratio a été calculé pour certaines variables et le seuil de signification fixé à $p \leq 0.05$ et IC à $95 \%$. L'analyse de la validité et de la fiabilité du questionnaire faite par le calcul du coefficient $\alpha$ de Cronbach, le nombre de dimensions du questionnaire avec une analyse de composantes principales (ACP), le coefficient de corrélation, le test de Bartlett et la mesure de précision de l'échantillon de Kaiser-Meyer-Olkin ont été calculés. 
Les résultats indiquent que $6 \%$ des malades hospitalisés étaient très satisfaits et $47 \%$ des patients satisfaits. Les patients qui avaient estimé qu'ils étaient bien accueillis, étaient satisfaits de manière globale à 56\% et $47 \%$ des patients ayant jugés l'accueil et le confort des chambres de mauvaise qualité au premier contact ont déclaré une insatisfaction. Cette observation indique que le premier contact est déterminant pour les patients. Par ailleurs, les résultats indiquent que pour plus de $70 \%$ des patients satisfaits, les aspects organisationnels, notamment, la gestion de file d'attente réduisant le temps d'attente, la communication de la démarche thérapeutique, la participation du patient dans la décision relative aux soins et l'individualisation des soins ont été déterminants $(p=0.000)$. En plus, la politique d'amélioration continue a induit des coûts et la hausse de prix, mais en revanche, les demandes des soins par les patients sont restées insensibles à cette hausse des coûts et le niveau de satisfaction assez élevé pour les patients ayants bénéficié des soins dans ces services $(p=0.000)$. L'étude a révélé que la qualité pour le patient est un concept lié à sa satisfaction. L'amélioration continue donne un avantage compétitif pour les établissements des soins de santé et permet d'améliorer le niveau de satisfaction des patients et l'utilisation des services, l'un des leviers de la performance des services de santé.

Mots clés : Satisfaction, Patients, Levier de Performance, services de santé, Lubumbashi, RDC

\section{Introduction}

La défaillance du système et politique de santé en République Démocratique du Congo prépare le lit à l'affaiblissement des services de santé. La gouvernance hospitalière souffre d'une inefficacité sur le plan organisationnel et managérial. Le système de santé de la RDC, à l'instar des autres systèmes de pays à faible revenu mérite une mise en œuvre urgente des stratégies innovantes afin de le rendre performant. Les Cliniques Universitaires de Lubumbashi rendent des services aux patients et deux modes de payement des factures des soins sont pratiqués, à savoir, un paiement direct et/ou un paiement indirect par les tiers payants. Toutefois, la léthargie du système de santé et le désengagement des décideurs et détenteurs d'enjeux constituent un dilemme pour la bonne qualité des soins et la satisfaction des usagers. Le faible financement et de subvention du pouvoir public rendent les soins inaccessibles pour certaines catégories de la population et la prise en charge de la grande partie du coût des soins de santé par les malades constitue un lourd fardeau pour les clients "patients " qui du reste, deviennent de plus en plus exigeants et insatisfaits de la qualité des services reçus. L'absence de certaines compétences, la non permanence des médecins, le détournement des malades vers les structures privées, le cumul de fonction pour extra muros en sont des tares qui viennent se greffer davantage sur les maux qui alourdissent l'insatisfaction des clients. Alors que la satisfaction des patients est devenue une préoccupation centrale dans le cadre de l'amélioration continue de l'offre des soins de santé, de l'importance de la place accordée actuellement au malade et constitue l'un des leviers pour la performance des services de santé. $[11,12,13,14,17]$. Cette approche de la qualité des soins et satisfaction des patients ou clients, consommateurs des services de santé, se trouve

*Corresponding author's ORCID ID: 0000-0001-5575-2435 DOI: https://doi.org/10.14741/ijmcr/v.6.5.9 actuellement au centre de plusieurs débats et innovations $[18,19,20,21,22]$. A ce niveau, il est important de relever certaines observations faites par certains chercheurs à travers le monde.

Les auteurs indiquent qu'à I'hôpital Saint Damien en Haïti/ Port-au-Prince, $87,8 \%$ des patients hospitalisés étaient satisfaits de l'accueil et $80,2 \%$ satisfaits de la communication contre une certaine insatisfaction observée pour les autres aspects de la prise en charge en hospitalisation [1].

En France, une moyenne de $83 \pm 9 \%$ des patients hospitalisés étaient satisfaits de la relation patientsoignant, $84 \pm 10 \%$ satisfaits des interventions thérapeutiques et $88 \pm 8 \%$ satisfaits du fonctionnement du service, avec une satisfaction globale assez faible de $69 \%$ [2]. Toujours en France, une autre étude a indiqué un niveau de satisfaction globale excellente dans plus de 90\% des cas, les patients estimaient avoir été accueillis aimablement. Au service des urgences, $70 \%$ des patients estimaient avoir peu attendu, moins d'une demi-heure. Quant à l'information donnée par le médecin, elle était jugée satisfaisante par plus de $90 \%$ des patients [3]. En Australie, $84 \%$ des patients avaient classé la prise en charge globale comme "très bonne» ou excellente, contre seulement $3 \%$ qui avaient évalué leurs soins comme «équitables». Les patients qui avaient attendu une heure ou moins avaient évalué leur prise en charge globale comme "excellente» bien que cela n'avait pas atteint la signification statistique $(66 \%$ versus $53 \%, p=$ 0,063 ) et avaient des scores médians plus élevés de satisfaction par rapport à ceux qui avaient attendu plus longtemps $(p<0,0001)$. Après ajustement, l'étude avait démontré une association statistiquement significative entre le temps d'attente et la meilleure note de satisfaction globale (OR 0,53; IC95\% [0,37-0,75]; p $<0,001$ ) [4]. En Suisse, les hôpitaux (53 hôpitaux) avaient une moyenne de satisfaction des patients de $76 \%$. les femmes et les seniors étaient davantage satisfaits à $81 \%$ [5]. Au Maroc, une étude avait observé que $66 \%$ des 
bénéficiaires du Régime d'assistance médicale étaient satisfaits au Centre Hospitalier Régional al farabi d'oujda. la dimension "demande des soins avec dignité" était consentie par $59 \%$ de l'ensemble des répondants et par $69 \%$ des personnes ayant utilisé un certificat d'indigence [6]. Au Cameroun, une étude avait rapporté que moins de $20 \%$ des districts de santé faisaient référence aux bonnes pratiques de gouvernance [7].

En République Démocratique du Congo, la mesure de la satisfaction des patients reste et demeure encore une question centrale parce que les patients participent à plus de $50 \%$ des revenus au budget de fonctionnement des services de santé par le payement direct. Les subventions du pouvoir public sont très limitées et la participation des patients de plus en plus croissante [8]. Dans ces conditions, il est essentiel de cerner le problème et proposer de nouvelles stratégies permettant une solidarité humaine face au risque lié à la maladie; la dispensation des soins de santé de qualité, équitables et respectueux des valeurs de couverture sanitaire universelle; et permettant d'agir comme levier de la performance des services de santé $[8,9,10,16]$.

Par conséquent, les questions ci-dessous constituent la problématique de cette enquête: - Quels sont les déterminants de la satisfaction des patients hospitalisés? - Quel est le niveau de satisfaction des patients hospitalisés vis-à-vis de la qualité des soins ? Comment les formations sanitaires de la R. D. Congo en général et en particulier les Cliniques Universitaires de Lubumbashi peuvent-elles assurer les soins de santé de qualité ?

\section{Méthodes}

C'est une étude descriptive transversale, portant sur la satisfaction des patients hospitalisés, l'un des leviers pour la performance des services de santé : Contribution à l'évidence scientifique, Lubumbashi, République Démocratique du Congo, étude réalisée aux Cliniques Universitaires de Lubumbashi. La population d'étude était constituée de 423 patients hospitalisés, estimés statistiquement en utilisant la formule de Sturge, pour la période allant du 01-Juillet-2016 au 01-Juillet-2017. Les données ont été collectées par l'observation directe, l'interview structurée et un questionnaire préalablement préétabli et pré-testé, adressé aux malades. Le questionnaire a été élaboré selon le modèle CCECQA (Comité de Coordination de l'Evaluation Clinique et de la Qualité en Aquitaine) et COMPAQH (Coordination pour la Mesure de la Performance et l'Amélioration de la Qualité Hospitalière), à la suite d'un test de fiabilité du questionnaire. Il a été bien évidemment adapté au contexte qui est celui des Cliniques Universitaires de Lubumbashi, un centre hospitalier tertiaire et de référence. La méthode analytique et celle comparative ont servis pour l'analyse et l'interprétation des résultats. Les logiciels Excel 2013, Epi info 7 et SPSS 21 ont été utilisés. Le test t Student, le test de Levene et le test de
$\mathrm{Chi}^{2}$ ont permis la comparaison des moyennes, des variances et les proportions. L'Odds ratio a été calculé pour certaines variables et le seuil de signification fixé à $p$ $\leq 0.05$ et IC à $95 \%$. L'analyse de la validité et de la fiabilité du questionnaire faite par le calcul du coefficient $\alpha$ de Cronbach, le nombre de dimensions du questionnaire avec une analyse de composantes principales (ACP), le coefficient de corrélation, le test de Bartlett et la mesure de précision de l'échantillon de Kaiser-Meyer-Olkin ont été calculés. Le logiciel Xmind-8 avait servi pour la présentation de la figure de déterminants de l'insatisfaction des malades. Les statistiques hospitalières d'utilisation des services du temps précédent notre enquête ont servi comme base de sondage ainsi que pour déterminer le pas d'incrémentation ou pas de sondage ( $K=1.123 / 423$ d'où $\mathrm{K}=2.65=3$ ). Ce qui revient à dire que dans chaque service visité, après avoir choisi de manière aléatoire simple et à l'aide de la table des nombres aléatoires le premier patient à interviewer, nous avons utilisé systématiquement le pas de sondage de trois.

Un échantillon des malades hospitalisés en médecine interne homme, médecine interne femme, Chirurgie, Pédiatrie, Urgences/REA et Gynécologie-obstétrique et ayant déjà fait au moins $48 \mathrm{~h}$ le jour de notre passage et ayant accepté volontiers et manifesté un consentement libre et éclairé ont été inclus dans cette étude. Seuls les malades "échappés- belles", les patients dont l'issue était autre que le décès ou évadés au terme de l'enquête ont été inclus dans l'étude. Par contre, les patients n'ayant pas encore accomplis 48 heures d'hébergement étaient simplement exclus.

\section{Résultats}

Les résultats indiquent que $56 \%$ des patients étaient de sexe masculin contre $44 \%$ pour le sexe féminin. $17 \%$ des patients hospitalisés étaient traqués pour acheter les médicaments fournis par le personnel et $16 \%$ étaient détournés par les médecins pour terminer ou poursuivre leurs soins dans les structures privées. Globalement, $6 \%$ des malades hospitalisés sont très satisfaits contre $47 \%$ des satisfaits. Pour ce qui est de l'accueil : $9 \%$ de patients sont très satisfaits et $56 \%$ satisfaits. Concernant le confort des chambres : $2 \%$ de patients sont très satisfaits et $29 \%$ satisfaits; quant à l'hygiène des sanitaires: $30 \%$ de patients sont satisfaits. A propos de la permanence des médecins spécialistes: seuls $4 \%$ de patients sont très satisfaits et $37 \%$ satisfaits (Tableau I). En ce qui concerne la qualité des soins : seulement $5 \%$ de patients la jugent excellente, $3 \%$ très bonne et $52 \%$ bonne (Tableau II). Cependant, les malades qui étaient globalement satisfaits, en rapport avec l'aspect catégorie du personnel, avaient une satisfaction inférieure à $50 \%$ (Tableau III). Les résultats relatifs au niveau de satisfaction des patients montrent que les aspects organisationnels, notamment, la procédure de paie, la facilité d'accès au service, la gestion de file d'attente 
réduisant le temps d'attente, la communication de la démarche thérapeutique, la participation du patient dans la décision relative aux soins et l'individualisation des soins ont été déterminants $(p=0.023)$ et ont obtenu des seuils de plus de $70 \%$ des patients satisfaits au service de laboratoire, au sein duquel la démarche qualité est appliquée.

Sur le plan économique, les résultats indiquent que la politique d'amélioration continue a induit des coûts et la hausse de prix, mais en revanche, les demandes des soins par les patients sont restées insensibles à cette hausse des coûts et le niveau de satisfaction assez élevé pour les patients ayants bénéficié des soins dans ces services $(p=0.000)$.
L'association entre certains déterminants et la satisfaction globale avait révélé une différence statistiquement significative: les patients qui étaient satisfaits de l'accueil, étaient moyennement satisfaits à $56 \%(p<0,05)$ (Tableau IV); pour le confort des chambres : $74 \%(p<0,05)($ Tableau $V)$; à propos de l'hygiène des sanitaires : $63 \%(p<0,05)$ (Tableau VI); en rapport avec I'humanisation des soins : $63 \%$ (Tableau VII) ; et en ce qui concerne la qualité des soins: $59 \%(p<0,05)$ (Tableau VIII). L'insatisfaction décriée par les malades est représentée par le résultat qualitatif suivant:

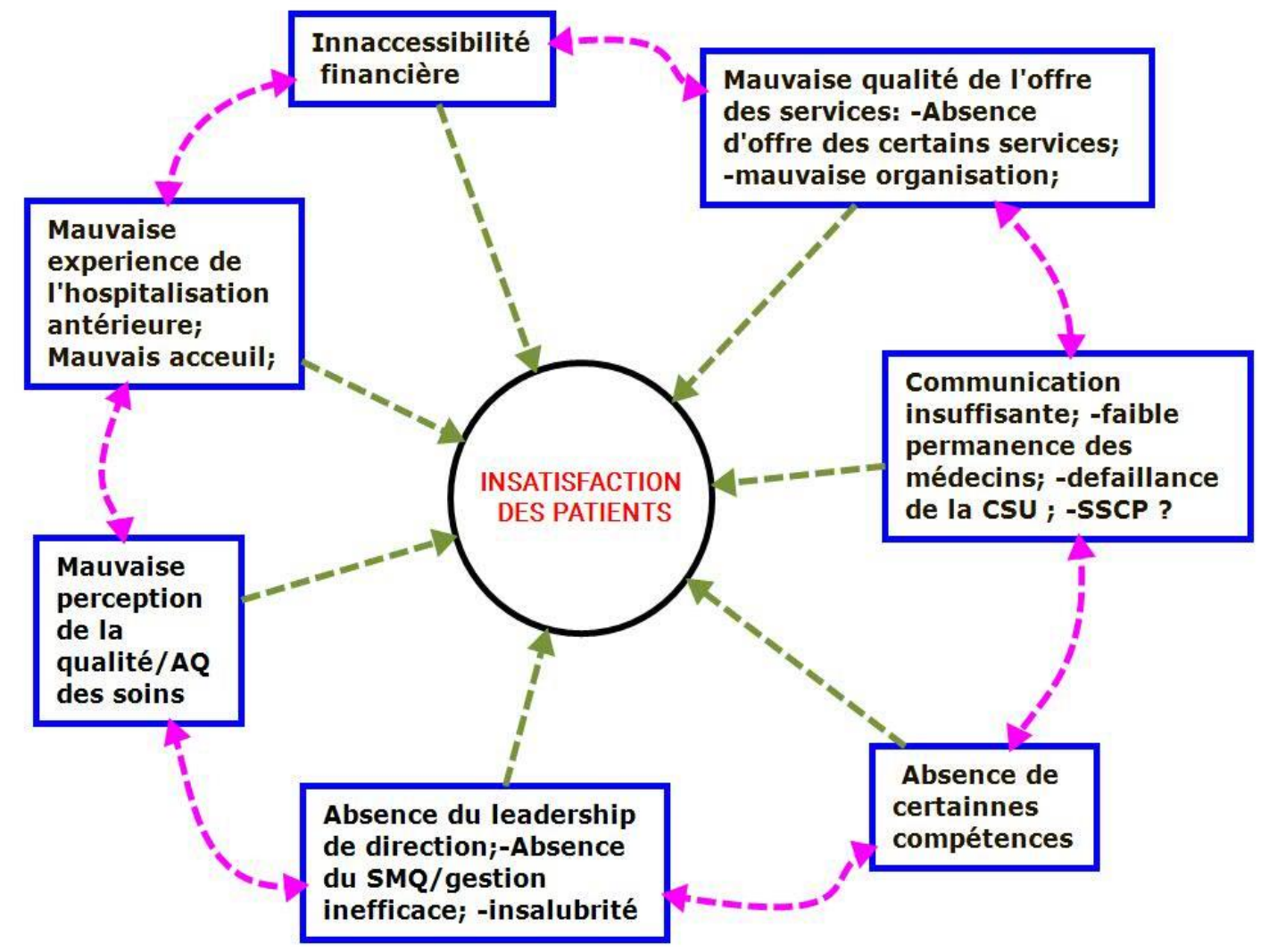

Figure 1 : Déterminants de l'insatisfaction vécue par les clients

L'insatisfaction des clients aux Cliniques Universitaires Lubumbashi
La liaison entre l'insatisfaction et ses facteurs
Les facteurs de l'insatisfaction
${ }^{*}$ LSU


Tableau I : Perception des patients hospitalisés en fonction des déterminants de la satisfaction

\begin{tabular}{|c|c|c|c|c|c|}
\hline \multirow{2}{*}{ Déterminants de satisfaction } & $\begin{array}{c}\text { Très } \\
\text { satisfaits }\end{array}$ & Satisfaits & Assez satisfaits & Peu satisfaits & Pas du tout satisfaits \\
\hline & $\%(n)$ & $\%(n)$ & $\%(n)$ & $\%(n)$ & $\%(n)$ \\
\hline Accueil & $9(38)$ & $56(236)$ & 28(119) & $1(5)$ & $6(25)$ \\
\hline Confort des chambres & $2(8)$ & $29(121)$ & $13(54)$ & $23(98)$ & $34(142)$ \\
\hline Hygiène des sanitaires & & $30(127)$ & $5(21)$ & $20(83)$ & $45(192)$ \\
\hline $\begin{array}{c}\text { Permanence des Médecins } \\
\text { généralistes }\end{array}$ & $8(32)$ & $47(198)$ & $22(94)$ & $13(56)$ & $10(43)$ \\
\hline $\begin{array}{c}\text { Permanence des Médecins } \\
\text { spécialistes }\end{array}$ & $4(17)$ & $37(157)$ & $24(102)$ & $21(89)$ & $14(58)$ \\
\hline Satisfaction globale & $6(26)$ & 47(198) & $27(116)$ & $14(59)$ & $6(24)$ \\
\hline
\end{tabular}

Tableau II: Evaluation de la qualité des services par les patients hospitalisés

\begin{tabular}{|c|c|c|c|c|c|c|}
\hline Qualité des services $(n=423)$ & Excellente & Très bonne & Bonne & Mauvaise & $\begin{array}{c}\text { Très } \\
\text { Mauvaise }\end{array}$ & $\begin{array}{c}\text { Ne sait } \\
\text { pas }\end{array}$ \\
\hline & $\%(n)$ & $\%(n)$ & $\%(n)$ & $\%(n)$ & $\%(n)$ & $\%(n)$ \\
\hline Prise en charge des inconforts & & & $57(242)$ & $43(181)$ & & \\
\hline Organisation de la sortie & & $14(57)$ & $50(211)$ & $31(132)$ & & $5(23)$ \\
\hline Qualité des soins & $5(20)$ & $3(12)$ & $52(221)$ & $39(166)$ & $1(4)$ & \\
\hline
\end{tabular}

Tableau III : Répartition des patients satisfaits globalement par rapport à la catégorie du personnel

\begin{tabular}{|c|c|c|}
\hline Catégorie du personnel & $\begin{array}{c}\text { Patients } \\
\text { satisfaits (n=224) }\end{array}$ & Pourcentage(\%) \\
\hline Sentinelles (Barrière entrée) & 91 & 40,6 \\
\hline Réceptionnistes & 98 & 43,8 \\
\hline Infirmières & 104 & 46,4 \\
\hline Médecins généralistes & 107 & 47,8 \\
\hline Médecins spécialistes & 88 & 39,3 \\
\hline Laborantins & 97 & 43,3 \\
\hline Pharmaciennes & 93 & 41,5 \\
\hline Personnel de surface & 82 & 36,6 \\
\hline
\end{tabular}

Tableau IV: Association entre l'accueil des patients et la satisfaction globale

\begin{tabular}{|c|c|c|c|c|}
\hline \multirow{2}{*}{ Accueil } & \multicolumn{2}{|c|}{ Satisfaction globale } & \multirow[b]{2}{*}{ Total(\%) } & \\
\hline & OUI (\%) & NON (\%) & & \\
\hline Bon accueil (oui) & $154(56)$ & $120(44)$ & $274(100)$ & \multirow{2}{*}{$p=0,0354$} \\
\hline Mauvais accueil (non) & $70(47)$ & $79(53)$ & 149(100) & \\
\hline
\end{tabular}

Tableau V : Association entre le confort des chambres des hospitalisés et la satisfaction globale

\begin{tabular}{|c|c|c|c|c|}
\hline \multirow{2}{*}{ Confort des chambres } & \multicolumn{2}{|c|}{ Satisfaction globale } & \multirow{2}{*}{ OR : 3,4405 } \\
\cline { 2 - 4 } & OUI (\%) & NON (\%) & Total(\%) \\
{$[2,1647 ; 5,4682]$}
\end{tabular}


Tableau VI: Association entre l’hygiène des sanitaires et la satisfaction globale des patients hospitalisés

\begin{tabular}{|c|c|c|c|c|}
\hline \multirow{2}{*}{ Hygiène des sanitaires } & \multicolumn{2}{|c|}{ Satisfaction globale } & & OR : 1,7967 \\
\cline { 2 - 4 } & OUI (\%) & NON (\%) & Total(\%) & $\begin{array}{c}\text { IC } \% \\
{[1,1731 ; 2,7517]}\end{array}$ \\
\hline Hygiène des sanitaires (oui) & $80(63)$ & $47(37)$ & $127(100)$ & $p=0,003$ \\
\hline Pas d'hygiène des sanitaires (non) & $144(49)$ & $152(51)$ & $296(100)$ & \\
\hline
\end{tabular}

Tableau VII: Association entre I'humanisation des soins des patients et la satisfaction globale

\begin{tabular}{|c|c|c|c|c|}
\hline \multirow{2}{*}{ Humanisation des soins } & \multicolumn{2}{|c|}{ Satisfaction globale } & \multirow{2}{*}{$\begin{array}{c}\text { OR }: 3,1038 \\
\text { IC } 95 \% \\
{[2,0556 ; 4,6865]}\end{array}$} \\
\cline { 2 - 4 } & OUI (\%) & NON (\%) & Total(\%) & \\
\hline $\begin{array}{c}\text { Humanisation des soins } \\
\text { (oui) }\end{array}$ & $169(63)$ & $99(37)$ & $268(100)$ & \\
\hline $\begin{array}{c}\text { Pas d'humanisation des } \\
\text { soins (non) }\end{array}$ & $55(35)$ & $100(65)$ & $155(100)$ & $\mathrm{p}=0,00000002$ \\
\hline
\end{tabular}

Tableau VIII : Association entre la qualité des soins réalisés et la satisfaction globale des malades hospitalisés

\begin{tabular}{|c|c|c|c|c|}
\hline \multirow{2}{*}{ Qualité des soins réalisés } & \multicolumn{2}{|c|}{ Satisfaction globale } & \multirow[b]{2}{*}{ Total(\%) } & \multirow{2}{*}{ OR : 2,5740} \\
\hline & OUI (\%) & NON (\%) & & \\
\hline Bonne qualité (oui) & 185(59) & $129(41)$ & $314(100)$ & \multirow{2}{*}{$\begin{array}{c}\text { IC95\% } \\
{[1,6387 ; 4,0433]} \\
p=0,00002\end{array}$} \\
\hline Mauvaise qualité (non) & $39(36)$ & $70(64)$ & $109(100)$ & \\
\hline
\end{tabular}

Tableau IX : Satisfaction des clients versus variables de gestion de la structure

\begin{tabular}{|c|c|c|c|c|c|c|}
\hline \multicolumn{7}{|c|}{ Satisfaction $(n=126)$} \\
\hline Variables & Oui & Non & Total & OR (IC 95\%) & $p$ & Decision \\
\hline \multicolumn{7}{|c|}{ Accueil et écoute } \\
\hline Mediocre & $4(3,2 \%)$ & $3(2,4 \%)$ & $7(5,6 \%)$ & $0,02(0,000-0,24)$ & 0.001 & $\mathrm{~S}$ \\
\hline Bonne & $117(92,8 \%)$ & $2(1,6 \%)$ & $119(94,4 \%)$ & & & \\
\hline \multicolumn{7}{|c|}{ Coûts de soins } \\
\hline Abordable & $98(77,8 \%)$ & $4(3,2 \%)$ & $102(85,7 \%)$ & $1,07(0,91-1,10)$ & 0.659 & NS \\
\hline Non abordable (Cher) & $23(18,3 \%)$ & $1(0,8 \%)$ & $24(14,3 \%)$ & & & \\
\hline \multicolumn{7}{|c|}{ Disponibilité du personnel } \\
\hline Oui & $106(84,1 \%)$ & $2(1,6 \%)$ & $108(85,7 \%)$ & $10,60(1,29-100,8)$ & 0.021 & $\mathrm{~S}$ \\
\hline Non & $15(11,9 \%)$ & $3(2,4 \%)$ & $18(14,3 \%)$ & & & \\
\hline \multicolumn{7}{|c|}{ Disponibilité des matériels } \\
\hline Oui & $112(88,9 \%)$ & $5(4 \%)$ & $117(92,9 \%)$ & $0,00(0,000-17,5)$ & 1 & NS \\
\hline Non & $9(7,1 \%)$ & $0(0 \%)$ & $9(7,1 \%)$ & & & \\
\hline \multicolumn{7}{|c|}{ Durée des soins observée } \\
\hline Non acceptable & $5(4 \%)$ & $5(4 \%)$ & $10(7,9 \%)$ & $0,00(0,00-0,07)$ & 0.000001 & $\mathrm{~S}$ \\
\hline Acceptable & $116(92,1 \%)$ & $0(0 \%)$ & $116(92,1 \%)$ & & & \\
\hline \multicolumn{7}{|c|}{ Caisse centralisée } \\
\hline Oui & $101(80,2 \%)$ & $2(1,6 \%)$ & $103(81,7 \%)$ & $7,57(0,94-70,32)$ & 0.042 & $S$ \\
\hline Non & $20(15,9 \%)$ & $3(2,4 \%)$ & $23(18,3 \%)$ & & & \\
\hline
\end{tabular}

La gestion du service par l'approche d'amélioration continue entraîne des coûts de la gestion qualité des services et une augmentation des coûts de soins de santé. Pour les demandes des patients par rapport aux fluctuations des coûts, les écarts positifs des prix l'emportent (test $t$ d'égalité des moyennes, $p=0.0000$ ) et dans l'hypothèse de variances inégales (test de levene, $p$ $=0.0000)$. Ces résultats indiquent que la demande 
d'analyses par la patientèle a été insensible à la hausse de prix induite par la hausse des coûts.

\section{Discussions des résultats}

L'étude a été menée auprès de 423 patients dont $56 \%$ étaient de sexe masculin contre $44 \%$ pour le féminin. Ce qui diffère de celui qui a été trouvé en Haïti/Port-auPrince, hôpital saint Damien (plus de $80 \%$ pour le sexe féminin et $20 \%$ pour le masculin) [1]. Leur étude prenait en compte les malades hospitalisés et les malades ambulatoires. C'est également diffèrent de celui observé en Australie (61\% pour le sexe masculin et $39 \%$ pour le féminin) [4]. Population non similaire et l'écart serait dû à la périodicité de notre enquête, aux malades hébergés dans les services pendant notre investigation et des types d'hôpitaux, lieu d'enquête pour les autres auteurs. Notre étude a démontré que $17 \%$ des patients hospitalisés étaient traqués pour acheter les médicaments fournis en informel par le personnel et $16 \%$ étaient sollicités et drainés par les médecins pour terminer ou poursuivre leurs soins dans les structures privées. C'est le détournement des malades, phénomène stigmatisé au service de gynéco-obstétrique et de la chirurgie ; et qui est commun dans les hôpitaux de pays à faible revenu, où les malades représentent une source potentielle des recettes.

Ce résultat est similaire à celui qui a été observé au Cameroun (dessous de table, paiements informels, vente illicite des médicaments, la surfacturation, la corruption à l'hôpital public, le racket, l'extorsion des fonds par intimidation, terreur ou chantage, les paiements officieux dans les services publics et moins de $20 \%$ des districts sanitaires faisaient recours aux pratiques de la bonne gouvernance) [7]. Ce phénomène pourrait s'expliquer par le fait que le système de santé Camerounais semble être proche de celui de la RD Congo et le contexte de gouvernance hospitalière et les pratiques Camerounaises en milieu hospitalier restent et demeurent encore très proches. C'est dans le contexte des pays en phase d'élaboration d'instructions et le pouvoir de subvention et de rémunération du personnel très bas. Notre étude a rapporté que $56 \%$ des patients étaient satisfaits de l'accueil et $9 \%$ très satisfaits. Pour la qualité des soins, seulement $5 \%$ de patients la jugent excellente, $3 \%$ très bonne et $52 \%$ bonne. Ces résultats sont faibles au regard de ce qui a été observé par d'autres chercheurs $(87,8 \%)$ $[1,2,3,4]$. Cette différence est due au fait que sous d'autres cieux, d'une part, le malade est considéré comme un client et la place lui accordée tient compte de ses exigences, ses attentes et son choix. Le client est " roi » dit-on. Ce qui est un tabou dans le contexte de la RD Congo. D'autre part, il s'agit d'un hôpital où la notion de la qualité des soins est de rigueur et constitue un atout. L'étude a indiqué que par rapport au confort des chambres, seulement $2 \%$ des patients sont très satisfaits, $29 \%$ satisfaits. Pour l'hygiène des sanitaires (installations sanitaires et Douches) $30 \%$ de patients sont satisfaits. Ce qui corrobore le résultat trouvé en France (15\% à 50\%) [3] mais opposable à celui observé en Haïti (>90\%) [1]. II a été noté que certains malades avaient clamé et dénoncé les odeurs nauséabondes dans les chambres, les douches et les installations sanitaires, les lits défectueux, mousses/matelas avec présence des urines ou du sang, l'absence de moustiquaires dans cette zone tropicale, l'absence de bonnes couvertures, l'absence de la réfection des lits. Ceci indique une inattention de l'administration hospitalière sur l'amélioration des conditions hygiéniques et le confort du patient. Notre recherche a révélé, pour ce qui est de la satisfaction par catégorie du personnel, des résultats inférieurs à $50 \%$. Les patients avaient clamé et décrié une faible permanence des médecins, manque d'empathie, qui du reste, sont souvent dans l'extra muros.

Quant à la satisfaction globale (optimale), nous avons observé que les patients qui sont très satisfaits avaient représenté seulement $6 \%$ et satisfaits $47 \%$. Ce qui est opposable aux résultats observés au Maroc, au Centre Hospitalier Régional al farabi d'oujda (69\%), en France $(64,5 \%$ et $69 \%)$ en Haïti $(>80 \%)$, en Suisse $( \pm 76 \%(66 \%$ $87 \%)[1,2,5,6]$.

Cette différence est due au système de management hospitalier, au mode de gestion, les pratiques de bonne gouvernance hospitalière, le non recours aux référentiels et non accréditation des hôpitaux en RD Congo.

\section{Limites de l'étude}

L'évaluation de la satisfaction des malades hospitalisés est limitée dans cette dissertation sur les aspects de la qualité des soins et de la satisfaction globale. La partie focalisée sur les déterminants de la satisfaction des patients en hospitalisation n'aborde pas de manière exhaustive tous les facteurs associés à la satisfaction des malades.

\section{Conclusion}

L'étude a révélé que la qualité pour le patient est un concept lié à sa satisfaction. L'amélioration continue donne un avantage compétitif pour les établissements des soins de santé et permet d'améliorer le niveau de satisfaction des patients et l'utilisation des services, l'un des leviers de la performance des services de santé. Le niveau de satisfaction est assez élevé pour les patients ayant bénéficié des soins dans les services pratiquant la politique d'amélioration continue versus les services offrant les soins standards. Les politiques de santé qui visent à améliorer la performance des services de santé devront aligner la satisfaction des patients comme l'un des éléments clés dans leur conception et leur mise en œuvre. 


\section{Conflit d'intérêt}

Les auteurs ne déclarent aucun conflit d'intérêt.

\section{Références}

[1]. Anna Cassandre Eustache, Anthony Monfiston, Cécile Tamara Georges, Johnny C., Michaelle Ericka Laforest (2010). Etude de la satisfaction de la clientèle par rapport à I'Hôpital Saint Damien en Haïti.

[2]. Ijaouane Elisabeth (2012). Evaluation de la satisfaction de soins en pédopsychiatrie par les enfants hospitalisés et par leurs parents, France.

[3]. Lopez A., Louis Remy, P. (2007). Mesure de la satisfaction des usagers des établissements de santé ( $n^{\circ} \mathrm{RM} 2007-$ 045P), France.

[4]. Dinh M. M., Enright, N., Walker, A., Parameswaran, A., Chu, M. (2012). Determinants of patient satisfaction in an Australian emergency department fast-track setting.

[5]. Eisler R. (2007). La satisfaction des patients hospitalisés en Suisse.

[6]. Mbarki, F.Z. (2014). Mesure de la satisfaction des patients bénéficiaires du RAMED hospitalisés au chr al farabi d'oujda vis-à-vis dudit régime.

[7]. Robert M., Ongolo-Zogo P., David Yondo, René OwonaEssomba (2010). Stimuler l'engagement des parties prenantes pour améliorer la gouvernance et accélérer la viabilisation des districts de santé au Cameroun Note d'information stratégique.

[8]. Henri Mundongo T., Didier Van Caillie, Frank Nduu N., Francis Mutach K., Françoise Malonga K., Grevisse Ditend Y., Pascal Tshimwang N., (2014).Risk of death and the economic accessibility at the dialysis therapy for the renal insufficient patients in Lubumbashi city, Democratic Republic of Congo.

[9]. Communauté de Pratique et prestation des services de santé, HHA, Fond Français Muskoka, Coopération belge au développement, OOAS (2013). Rapport de la Conférence Régionale "Les districts sanitaires en Afrique : Progrès et perspectives 25 ans après la déclaration d'Harare ", Dakar, Sénégal.
[10].Crommelynck A., Degraeve, K., Lefèbvre, D. (2013). L'organisation et le financement des hôpitaux.

[11]. Bruno Rachel Frédérique, Doucet Jean Gary, Merisier Elvis, Metayer Linda (2009). Etude de la satisfaction de la clientèle à l'hôpital de fort-liberté, en Haïti.

[12]. Daniel, P., Perneger, T., Burnand, B., Fabrizio, B., Nicolas (2000). La qualité de soins dans les établissements hospitaliers suisses : analyse de six indicateurs.

[13].Guisset A.-L., Sicotte C., Leclercq Pol, D'Hoore William (2015). Définition de la performance hospitalière: une enquête auprès des divers acteurs stratégiques au sein des hôpitaux.

[14]. Johan Van der Heyden (2008). Satisfaction du patient.

[15]. Delvosalle Christian (2004). Normes et Référentiels Qualité.

[16]. Pawan K., Abdul Majeed Khan (2016). Développent of Job Satisfaction Scale for Health Care Providers.

[17]. Nguyen Thi, P.L., T.G. Lê, Empereur F., Briançon S. (2002). Satisfaction des patients hospitalisés à hô chi minhville, Viet Nam.

[18]. Dely, C., Pascal, J., Barjonet, L., Levasseur, G., \& Lombrail, P. (2009). Les représentations de patients adhérents à un réseau de santé: perspectives d'amélioration de leur prise en charge. La Presse Medicale, 38(2), 185-192.

[19]. Bouillon, M. L., Ferrier, G. D., Stuebs Jr, M. T., \& West, T. D. (2006). The economic benefit of goal congruence and implications for management control systems. Journal of Accounting and Public Policy, 25(3), 265-298.

[20]. Beckett, A., Hewer, P., \& Howcroft, B. (2000). An exposition of consumer behaviour in the financial services industry. International Journal of Bank Marketing, 18(1), 15-26.

[21]. Banker, R. D., Bardhan, I. R., \& Chen, T. Y. (2008). The role of manufacturing practices in mediating the impact of activity-based costing on plant performance. Accounting, organizations and society, 33(1), 1-19.

[22].Ang, L., \& Buttle, F. (2006). Customer retention management processes: A quantitative study. European Journal of Marketing, 40(1/2), 83-99. 